\title{
EXACT UPPER BOUND ON THE SUM OF SQUARED NEAREST-NEIGHBOR DISTANCES BETWEEN POINTS IN A RECTANGLE
}

\author{
IOSIF PINELIS
}

Abstract. An exact upper bound on the sum of squared nearest-neighbor distances between points in a rectangle is given.

Mathematics subject classification (2010): 26B25, 26D20, 49K30, 51M16, 52A40, 52A41.

Keywords and phrases: Exact bounds, metric geometry, geometric inequalities, nearest-neighbor distances, convexity, non-convex optimization.

\section{REFERENCES}

[1] P. K. Agarwal, L. ARGE, AND F. STAALs, Improved dynamic geodesic nearest neighbor searching in a simple polygon, In 34th International Symposium on Computational Geometry, volume 99 of LIPIcs. Leibniz Int. Proc. Inform., pages Art. No. 4, 14. Schloss Dagstuhl. Leibniz-Zent. Inform., Wadern, 2018

[2] A. Andoni, A. Naor, A. Nikolov, I. Razenshteyn, And E. Waingarten, Hölder homeomorphisms and approximate nearest neighbors, In 59th Annual IEEE Symposium on Foundations of Computer Science-FOCS 2018, pages 159-169. IEEE Computer Soc., Los Alamitos, CA, 2018.

[3] F. AUREnhammer, Voronoi diagrams - a survey of a fundamental geometric data structure, ACM Comput. Surv., 23(3):345-405, Sept. 1991.

[4] S. Basu, R. Pollack, AND M.-F. Roy, Algorithms in real algebraic geometry, volume 10 of Algorithms and Computation in Mathematics, Springer-Verlag, Berlin, second edition, 2006.

[5] T. B. Berrett, R. J. Samworth, And M. Yuan, Efficient multivariate entropy estimation via k-nearest neighbour distances, Ann. Statist., 47(1):288-318, 2019.

[6] W. GaO, S. OH, AND P. VIS WANATH, Demystifying fixed k-nearest neighbor information estimators, IEEE Trans. Inform. Theory, 64(8):5629-5661, 2018.

[7] R. JANSSEn, M. Jones, P. L. ERdős, L. VAn IERSEL, And C. Scornavacca, Exploring the tiers of rooted phylogenetic network space using tail moves, Bull. Math. Biol., 80(8):2177-2208, 2018.

[8] MathOVerflow, Sum of squared nearest-neighbor distances between points in a square, 2019, https://mathoverflow.net/questions/321033/sum-of-squared-nearest-neighbor -distances-between-points-in-a-square/321048\#321048. 